\title{
Multistability Analysis, Coexisting Multiple Attractors, and FPGA Implementation of Yu-Wang Four-Wing Chaotic System
}

\author{
Fei Yu $\left(\mathbb{D},{ }^{1,2}\right.$ Li Liu $\left(\mathbb{D},{ }^{1}\right.$ Hui Shen $\left(\mathbb{D},{ }^{1}\right.$ Zinan Zhang $\mathbb{D D}^{1}$ Yuanyuan Huang $\mathbb{D}^{1},{ }^{1}$ Shuo Cai ${ }^{1}{ }^{1}$ \\ Zelin Deng $\mathbb{D}^{1}{ }^{1}$ and Qiuzhen Wan $\mathbb{D}^{3}$ \\ ${ }^{1}$ School of Computer and Communication Engineering, Changsha University of Science and Technology, Changsha 410114, China \\ ${ }^{2}$ Guangxi Key Laboratory of Cryptography and Information Security, Guilin University of Electronic Technology, Guilin 541004, \\ China \\ ${ }^{3}$ Hunan Provincial Key Laboratory of Intelligent Computing and Language Information Processing, Hunan Normal University, \\ Changsha 410081, China
}

Correspondence should be addressed to Fei Yu; yufeiyfyf@csust.edu.cn and Qiuzhen Wan; wanqiuzhen@sina.com

Received 15 June 2020; Revised 10 July 2020; Accepted 13 July 2020; Published 29 August 2020

Guest Editor: Viet-Thanh Pham

Copyright ( 2020 Fei Yu et al. This is an open access article distributed under the Creative Commons Attribution License, which permits unrestricted use, distribution, and reproduction in any medium, provided the original work is properly cited.

\begin{abstract}
In this paper, we further study the dynamic characteristics of the Yu-Wang chaotic system obtained by Yu and Wang in 2012. The system can show a four-wing chaotic attractor in any direction, including all 3D spaces and 2D planes. For this reason, our interest is focused on multistability generation and chaotic FPGA implementation. The stability analysis, bifurcation diagram, basin of attraction, and Lyapunov exponent spectrum are given as the methods to analyze the dynamic behavior of this system. The analyses show that each system parameter has different coexistence phenomena including coexisting chaotic, coexisting stable node, and coexisting limit cycle. Some remarkable features of the system are that it can generate transient one-wing chaos, transient two-wing chaos, and offset boosting. These phenomena have not been found in previous studies of the Yu-Wang chaotic system, so they are worth sharing. Then, the RK4 algorithm of the Verilog 32-bit floating-point standard format is used to realize the autonomous multistable 4D Yu-Wang chaotic system on FPGA, so that it can be applied in embedded engineering based on chaos. Experiments show that the maximum operating frequency of the Yu-Wang chaotic oscillator designed based on FPGA is $161.212 \mathrm{MHz}$.
\end{abstract}

\section{Introduction}

Due to the extreme sensitivity of chaos to initial conditions and parameters and the characteristics of the generated chaotic sequence such as aperiodicity and pseudo-randomicity, chaos has been widely used in memristors [1-4], random number generators [5-7], electronic circuits [8-10], image encryption [11-14], complex networks [15-19], synchronization [20-23], and secure communication [24-28] in the past two decades. Up to now, numerous various chaotic systems have been extensively studied, including multiwing chaotic systems [29, 30], multiscroll chaotic systems [31-34], hyperchaotic systems [35-38], and chaotic systems with hidden attractors [39-42].
A class of chaotic systems are characterized by the coexistence of many different types of attractors, a phenomenon referred to as multistability which has become a very important research topic and received much attention recently [43-46]. In [44], a smooth piecewise quadratic nonlinear four-wing chaotic system is proposed. When the appropriate parameters including a two-wing and four-wing chaotic attractor are selected, the system can observe four kinds of unconnected coexisting stable states under different initial values and show rich dynamic behaviors. In [45], a 4D chaotic system with planar equilibrium is introduced. Through dynamic analysis, it is revealed that the chaotic system has three different types of coexisting attractors in a certain parameter range: chaotic attractors, stable node 
attractors, and limit cycle attractors. In addition, when the initial conditions of a state variable change and the number of attractors approaches infinity, this phenomenon is called extreme multistability [47-50]. More and more attention has been paid to the research related to extreme multistability. In [47], the flux-controlled memristor model is introduced into the existing 5D hyperchaotic autonomous system, and a 6D autonomous system is obtained. By analyzing the dynamics of the system, it is found that the system has the characteristics of extreme multistability, bursting, transient chaos, and offset boosting phenomenon.

Chaos generator is one of the most basic structures of chaos in practical application. Chaos generator is mainly realized by analog and digital methods. The method to realize a chaotic system in the analog circuit is mainly by using discrete components on the combined bread board $[30,32,42]$. The chaotic signal realized by this method is unstable, and its portability is very poor. Secondly, the integrated circuit (IC) chaos generator chip is designed by the CMOS technology, and the chaos generator based on IC can achieve the highest performance [51-53]. However, the implementation based on IC is not easy to realize the frequency tunability of the chaotic oscillator, and there are some problems such as a long design period and a high cost of the tape [54-56]. Compared with analog generators, chaos generators based on digital circuits have comparative advantages. Digital chaos generators are implemented by the digital signal processor (DSP) $[57,58]$ and the field programmable gate array (FPGA) [59-65]. In complex mathematical operations, DSP chips generally perform operations sequentially, so it takes a long time to calculate output values sequentially using DSP-based chaos generators. On the contrary, FPGA chips have a relatively flexible architecture that allows simultaneous computation, and FPGA chip design and test cycle costs are particularly low [66-68]. Through the numerical and reconfigurable characteristics of FPGA, the FPGA-based chaos generator and its application can be more flexible.

In this study, we consider the dynamics of a relatively simple chaotic system with a fully qualified four-wing chaotic attractor introduced by $\mathrm{Yu}$ et al. [69], with particular emphasis on the chaos mechanism and the possibility of multiple coexisting attractors varying with five system parameters. Chaotic systems with coexisting attractors have great potential for engineering applications. The research on the multistability of the Yu-Wang system has not been fully discussed, which is worthy of further study. In this paper, we find and focus on the multistability window. Secondly, some remarkable characteristics of the system such as transient chaos and offset boosting can be generated under certain conditions.

The rest of this paper is organized as follows. The mathematical model of the $\mathrm{Yu}$-Wang chaotic system is described and analyzed in Section 2. In Section 3, we analyze the dynamic characteristics of the system in detail. The complex dynamics of the system is analyzed from five aspects: coexistence of attractors with different parameters, multistability, basin of attraction, transient chaos, and offset boosting. In Section 4, we use the RK4 algorithm and the
Verilog language to design a Yu-Wang chaotic oscillator based on FPGA on Vivado 2018.3. Then, by analyzing the statistical data of the FPGA chip, the simulation results and the phase diagram of the oscilloscope output are obtained. Finally, some conclusions are given in Section 5.

\section{Description and Analysis of the Model of Yu-Wang Chaotic System}

The $\mathrm{Yu}-$ Wang chaotic system, which was proposed by $\mathrm{Yu}$ et al. [69], is described by the following simple 4D autonomous system with four quadratic nonlinear terms:

$$
\left\{\begin{array}{l}
\dot{x}=-a x+y z+b w \\
\dot{y}=c y-x z \\
\dot{z}=x y-d z \\
\dot{w}=x z-e w
\end{array}\right.
$$

where $x, y, z$, and $w$ are the state variables of the system and $a, b, c, d$, and $e$ are the system parameters. When $a=8$, $b=5, c=12, d=60$, and $e=4$ and the initial conditions are $[2,1,1,2]$, the system is a fully four-wing chaotic system. It is very interesting that the attractors of the system can exhibit a four-wing form in any direction of any phase plane, including all 3D spaces and 2D planes as shown in Figure 1. The four Lyapunov exponents (LEs) of system (1) are LE1 = 1.4247, LE2 $=0.0014$, LE3 $=-6.4991$, and LE4 $=-54.9120$, respectively, and the system has positive Lyapunov exponents, so the system has a very interesting dynamic behavior. The Yu-Wang system is symmetric about the $z$ axis; that is, it remains unchanged under the coordinate transformation $(x, y, z, w) \longrightarrow(-x,-y, z,-w)$.

The equilibrium point of system (1) can be obtained by solving the following equations:

$$
\left\{\begin{array}{l}
-a x+y z+b w=0 \\
c y-x z=0 \\
x y-d z=0 \\
x z-e w=0
\end{array}\right.
$$

On the basis of equation (2), it is easy to get $x= \pm \sqrt{c d}$. The following two cases are discussed:

(i) When $x=\sqrt{c d}$, substituting $x$ into equation (2), we get

$$
\left\{\begin{array}{l}
-a \sqrt{c d}+y z+b w=0 \\
c y-\sqrt{c d} z=0 \\
\sqrt{c d} y-d z=0 \\
\sqrt{c d} z-e w=0
\end{array}\right.
$$

and according to equation (3), we obtain the following equation:

$$
z_{1,2}=\frac{-(b c / e) \pm \sqrt{\left(b^{2} c^{2} / e^{2}\right)+4 a c}}{2}
$$




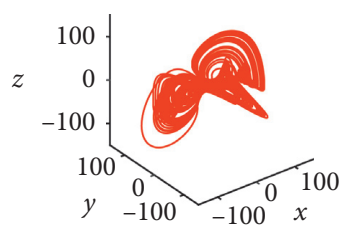

(a)

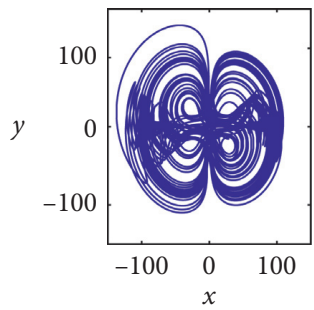

(d)

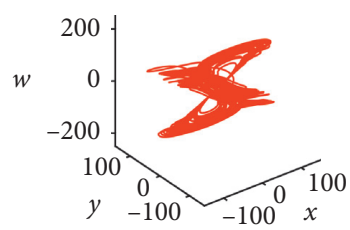

(b)

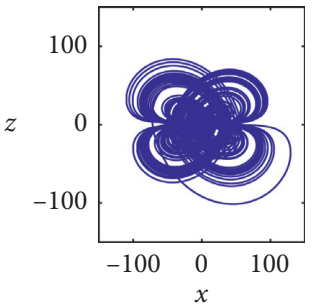

(e)

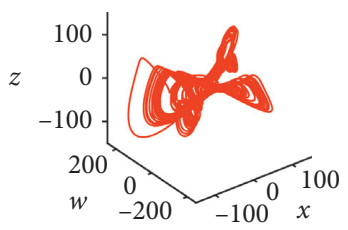

(c)

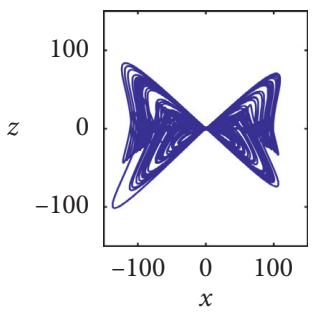

(f)

FIGURE 1: The four-wing chaotic attractor of system (1) in 3D spaces and 2D planes: (a) in the $x-y-z$ space, (b) in the $x-y-w$ space, (c) in the $x-w-z$ space, (d) in the $x-y$ plane, (e) in the $y-z$ plane, and (f) in the $x-z$ plane.

and because $y=\sqrt{(d / c)} z$ and $w=(\sqrt{c d} / e) z$, we can get

$$
\begin{aligned}
& y_{1,2}=\frac{-(b c / e) \pm \sqrt{\left(b^{2} c^{2} / e^{2}\right)+4 a c}}{2 \sqrt{c / d}} \\
& w_{1,2}=\frac{-(b c / e) \pm \sqrt{\left(b^{2} c^{2} / e^{2}\right)+4 a c}}{2 e / \sqrt{c d}}
\end{aligned}
$$

(ii) When $x=-\sqrt{c d}$, substituting $x$ into equation (2), we get

$$
\left\{\begin{array}{l}
a \sqrt{c d}+y z+b w=0, \\
c y+\sqrt{c d} z=0, \\
-\sqrt{c d} y-d z=0, \\
-\sqrt{c d} z-e w=0
\end{array}\right.
$$

and based on equation (6), we can obtain

$$
\begin{aligned}
& z_{3,4}=-\frac{-(b c / e) \pm \sqrt{\left(b^{2} c^{2} / e^{2}\right)+4 a c}}{2}, \\
& y_{3,4}=-\frac{-(b c / e) \pm \sqrt{\left(b^{2} c^{2} / e^{2}\right)+4 a c}}{2 \sqrt{(c / d)}}, \\
& w_{3,4}=-\frac{-(b c / e) \pm \sqrt{\left(b^{2} c^{2} / e^{2}\right)+4 a c}}{2(e / \sqrt{c d})} .
\end{aligned}
$$

According to the above analysis, the five equilibrium points of system (1) are $S_{0}=[0,0,0,0], S_{1,2}=\left[\sqrt{c d}, y_{1,2}\right.$, $\left.z_{1,2}, w_{1,2}\right]$, and $S_{3,4}=\left[-\sqrt{c d}, y_{3,4}, z_{3,4}, w_{3,4}\right]$ when $a=8$, $b=5, c=12, d=60$, and $e=4$. The five equilibrium points of system (1) can be calculated as $S_{0}=[0,0,0,0], S_{1,3}=$ $[ \pm 26.83, \pm 10.82,4.84, \pm 32.47], \quad$ and $S_{2,4}=[ \pm 26.84$, $\mp 44.36,-19.84, \mp 133.09]$.
The Jacobian matrix of system (1) can be described as

$$
\mathrm{JO}=\left[\begin{array}{cccc}
-a & z & y & b \\
-z & c & -x & 0 \\
y & x & -d & 0 \\
z & 0 & x & -e
\end{array}\right] .
$$

In order to consider the stability of the system, each equilibrium point is considered to calculate the corresponding eigenvalue according to the Jacobian matrix, and the eigenvalues are, respectively, obtained as $(-8,12,-60,-4),(-51.65,-11.54,1.60+9.67 i, 1.60-9.67 i)$, $(-88.54,15.39+65.61 i, 15.39-65.61 i,-2.25), \quad(-51.65$, $-11.54,1.60+9.67 i, 1.60-9.67 i)$, and $(-88.54,15.39+$ $65.61 i, 15.39-65.61 i,-2.25)$. According to the five eigenvalues, it can be judged that system (1) has an unstable saddle focal equilibrium point, which will lead to chaos.

The dissipativity of system (1) is described as

$$
\nabla V=\frac{\mathrm{d} \dot{x}}{\mathrm{~d} x}+\frac{\mathrm{d} \dot{y}}{\mathrm{~d} y}+\frac{\mathrm{d} \dot{z}}{\mathrm{~d} z}+\frac{\mathrm{d} \dot{w}}{\mathrm{~d} w}=-a+c-d-e=-60 .
$$

Equation (9) shows that the divergence is negative. Consequently, the general condition of dissipativity associated with the existence of attractive sets in system (1) is fulfilled.

\section{Dynamic Characteristic Analysis of System (1)}

In this section, complex dynamics of the $\mathrm{Yu}-\mathrm{Wang}$ autonomous chaotic system is numerically investigated with the help of various tools. These include the bifurcation diagram, Lyapunov exponents, phase portraits, and attraction basins. All numerical simulations are based on the resolution of system (1) using the fourth-order Runge-Kutta method. For the computation of Lyapunov exponents, the algorithm proposed by Wolf et al. [70] is used. 
3.1. Dynamic Analysis of Different System Parameters. The dynamic behaviors of the parameters in system (1) are analyzed. It is found that there are many kinds of attractors coexisting in system (1). Table 1 shows the dynamic behavior of different parameters of the system.

3.1.1. Case 1. Fixing the parameters $b=5, c=12, d=60$, and $e=4$, we make the parameter $a$ change in the range of $(0,40]$. Figure $2(a)$ is the bifurcation diagram of the system in the initial conditions $[2,1,1,2]$ and $[-2,-1,1,-2]$, and Figure 2(b) is the largest LE spectrum. From Figure 2, we can see that the dynamic characteristics of the system are very complex, and there are many coexisting attractors. When the parameter $a \in(0,11.1)$, system (1) shows a chaotic state. When $a \in[11.1,12.6]$, system (1) is in a periodic state, and when $a=11.5, a=12.3$, and $a=12.5$, the attractors of system (1) are, respectively, represented by the one-wing period-1, one-wing period-2, and one-wing period-4, and the phase portraits of the coexisting attractors are shown in Figures 3(a)-3(c). When $a \in(12.6,19]$, the system behaves as a chaotic state. Especially when $a=12.6$, system (1) presents the one-wing chaotic state, and its coexisting attractor is shown in Figure 3(d). When $a=15.8$, the system is in the three-wing period state, and its coexisting attractor is shown in Figure 3(e). When $a \in(19,23.8)$, the inverse period bifurcations and period bifurcation occur in this system, which are generally consistent with $a \in[11.1,12.6]$. Therefore, the same phenomenon is not shown in Figure 3. When $a=20$, system (1) is in the four-wing period-1 state, and its coexistence attractor is shown in Figure 3(f). When $a=19.1$, system (1) is in the four-wing period- 4 state, and its coexistence attractor is shown in Figure $3(\mathrm{~g})$. When $a \in[23.8,31.5)$, the system shows a new chaotic state. When $a=28$, system (1) is a four-wing chaotic attractor, and its coexisting attractor is shown in Figure $3(\mathrm{~h})$. When $a \in[31.5,40]$, the system is in a stable state, and when $a=31.6$, system (1) is in a stable state, and the phase portrait of its coexistence point attractor is shown in Figure 3(i).

3.1.2. Case 2. Fixing the parameters $a=8, c=12, d=60$, and $e=4$, we make the parameter $b$ change in the range of $[-20,20]$. Figure $4(a)$ shows the bifurcation diagram of the system in the initial conditions $[2,1,1,2]$ and $[-2,-1,1,-2]$, and Figure 4(b) shows the largest LE spectrum. From Figure 4 , the system is completely symmetric with respect to the dynamic characteristics of parameter $b$. When parameter $b \in[0,2.3]$, system (1) has an inverse period bifurcation, in which when $b=0, b=0.5$, and $b=0.58$, system (1) is displayed as the one-wing period-1, one-wing period-2, and one-wing period-4, respectively, and the phase portraits of their coexisting attractors are shown in Figures 5(a)-5(c); when $b \in(2.3,20]$, the system (1) state is mostly chaotic, and some periodic windows are included; especially when $b=19.35$, the system is a two-wing period phenomenon, and its coexistence attractor is shown in Figure 5(d). When $b=17.8$, the system is in the three-wing periodic state, and its coexistence attractor is shown in Figure 5(e). When $b=15$, the system is in four-wing chaos, and its coexistence attractor is shown in Figure 5(f).

3.1.3. Case 3. Fixing the parameters $a=8, b=5, d=60$, and $e=4$, let the parameter $c$ change in the range of $[0,50]$. Figure 6(a) shows the bifurcation diagram of the system under two different initial conditions (the blue region represents the initial conditions of $[2,1,1,2]$ and the red region represents the initial conditions of $[-2,-1,1,-2])$. Figure 6(b) shows the largest LE exponent spectrum of Figure 6(a). According to Figure 6, the system has experienced the process from periodic state to chaos to periodic state to chaos to period doubling bifurcation to chaos. In the interval $c \in[0,10.4]$, system (1) is in the periodic state. When $c=0.06, c=0.1$, and $c=0.2$, the system is in the one-wing period-1, one-wing period-3, and two-wing period-3, respectively, and their coexisting attractor phase portraits are shown in Figures $7(\mathrm{a})-7(\mathrm{c})$. Figures $7(\mathrm{~d})$ and $7(\mathrm{e})$ are the phase portraits of the coexistence of attractors in two different states when the system changes from twowing multiperiod to two-wing period-1 state. In the interval $c \in(10.4,14.9)$, the system is in a chaotic state. When $c=10.6$, the system has a four-wing chaotic attractor, and its coexisting attractor is shown in Figure $7(\mathrm{f})$; in $c \in[14.9,15.2)$, the system is in the periodic state. When $c=15$, the system is in the twowing period-1 state, and its coexisting attractor is shown in Figure 7(g); the system is chaotic in the interval $c \in[15.2,29.4)$. When $c=15.32$, the system has a two-wing chaotic attractor, and its coexisting attractor is shown in Figure $7(\mathrm{~h})$; in the interval $c \in[29.4,32.1)$, system (1) experiences period doubling bifurcation, in which when $c=31, c=31.8$, and $c=31.9$, the system is in the one-wing period-1, one-wing period-2, and one-wing period-4 states, respectively, and the phase portraits of their coexisting attractors are shown in Figures $7(\mathrm{i})-7(\mathrm{k})$. In the interval $c \in[32.1,50]$, the system is in a chaotic state. When $c=32.5$, the system has a one-wing chaotic attractor, and its coexisting attractor is shown in Figure 7(l).

3.1.4. Case 4. For the given parameters $a=8, b=5, c=12$, and $e=4$, let parameter $d$ vary within the range of $[16,100]$. Figure $8(\mathrm{a})$ is the bifurcation diagram of the system under two different initial conditions (the blue region is the initial conditions of $[2,1,1,2]$ and the red region is the initial conditions of $[-2,-1,1,-2])$. Figure $8(\mathrm{~b})$ is the largest LE spectrum of Figure $8(\mathrm{a})$. As can be seen from Figure 8 , the system dynamics is very complex, with period doubling bifurcation, inverse period bifurcation, and chaotic states. When $d=16$, the system is in the period-1 state; when $d=17$, the system is in the period- 2 state; when $d=17.2$, the system is in the period- 4 state; when $d=17.8$, the system has one-wing chaotic attractors; when $d=25$, the system has four-wing chaotic attractors; when $d=42$, the system is in a multiperiod state; when $d=50$, the system is in a multiperiod state; when $d=68$, the system is in the two-wing period-1 state; when $d=70$, the system is in the two-wing multiperiod state. The phase portrait of coexisting attractors 
TABle 1: Dynamic behavior under different parameters.

\begin{tabular}{|c|c|c|}
\hline Case & Parameter value & Dynamic behavior \\
\hline Case 1 & $b=5, c=12, d=60, e=4, a \in(0,40]$ & $\begin{array}{l}\text { Three kinds of periodic phenomena, two kinds of chaotic } \\
\text { attractors with different wings and point attractor }\end{array}$ \\
\hline Case 2 & $a=8, c=12, d=60, e=4, b \in[-20,20]$ & $\begin{array}{c}\text { Three different types of periodic phenomena and } \\
\text { four-wing chaotic attractors }\end{array}$ \\
\hline Case 3 & $a=8, b=5, d=60, e=4, c \in[0,50]$ & $\begin{array}{l}\text { Seven kinds of periodic phenomena, two kinds of } \\
\text { quasiperiodic attractors, and two kinds of chaotic attractors }\end{array}$ \\
\hline Case 4 & $a=8, b=5, c=12, e=4, d \in[16,100]$ & $\begin{array}{l}\text { Three different types of periodic phenomena and } \\
\text { two different types of chaotic attractors }\end{array}$ \\
\hline Case 5 & $a=8, b=5, c=12, d=60, e \in[0,44]$ & $\begin{array}{l}\text { Two kinds of periodic phenomena and two kinds of } \\
\text { chaotic attractors with different wings }\end{array}$ \\
\hline
\end{tabular}

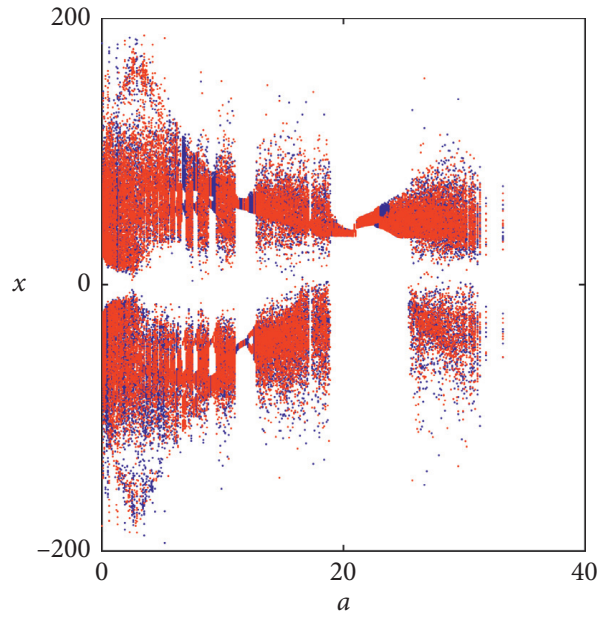

(a)

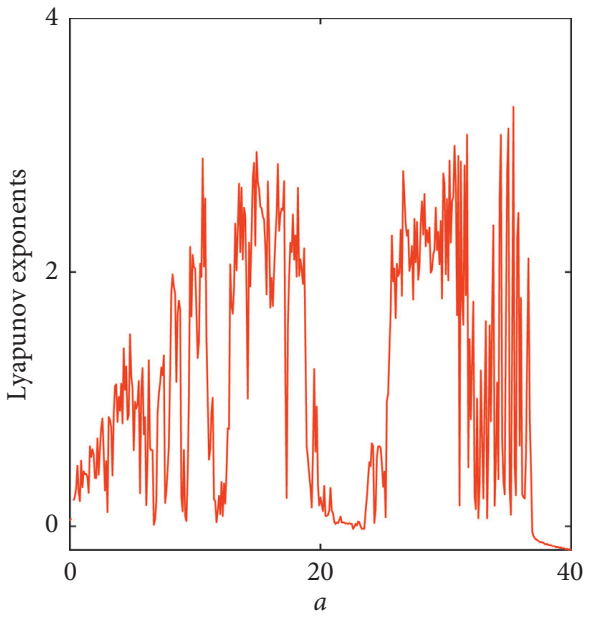

(b)

Figure 2: The bifurcation diagram and the largest LE for increasing parameter $a \in(0,40]$. (a) The bifurcation diagram with initial conditions $[2,1,1,2]$ (blue) and $[-2,-1,1,-2]$ (red). (b) The largest LE.

of the above analyzed states under two initial conditions is shown in Figures 9(a)-9(i), respectively.

3.1.5. Case 5. For the given parameters $a=8, b=5, c=12$, and $d=60$, the parameter $e$ changes in the range of $[0,44]$. Figure 10(a) shows the bifurcation of the system under two different initial conditions (the blue indicates that the initial condition is $[2,1,1,2]$ and red indicates that the initial condition is $[-2,-1,1,-2])$. Figure 10 (b) shows the largest LE spectrum of Figure 10(a). As can be seen from Figure 10, the system undergoes the process of period doubling bifurcation to chaos to period doubling bifurcation to chaos to periodic state. In the interval $e \in[0,2.5]$, system (1) experiences period doubling bifurcation. When $e=0.2$, the system is in the four-wing periodic state, and Figure 11(a) is the phase portrait of its coexisting attractor. When $e=1.5, e=2$, and $e=2.1$, the system presents the one-wing period-1, one-wing period-2, and one-wing period-4, respectively, and the phase portraits of their coexisting attractors are shown in Figures 11(b)-11(d); in $e \in(2.5,11.6]$, the system is in a chaotic state. Figure $11(\mathrm{e})$ shows the phase portraits of the coexistence attractor when $e=5$; in the interval $e \in(11.6,15.2)$, the system experiences period doubling bifurcation, and its phase portrait is the same as that of the previous period doubling bifurcation; in $e \in[15.2,42.3]$, the system is in a chaotic state. When $e=17$, the system has a two-wing chaotic attractor, whose coexisting attractor is shown in Figure 11(f).

3.2. Multistability. Coexistence of multiple attractors is a very interesting phenomenon in nonlinear systems. It only changes the initial conditions, and the system can produce multiple attractor coexistence. Multistability is the inherent property of many nonlinear dynamical systems and is the result of coexistence of many attractors. In short, a system is multistable if it has multiple attractors, and the external excitation is strong enough to switch between stable states. In this section, we will study the multistability of the $4 \mathrm{D}$ Yu-Wang chaotic system.

Figure $12(\mathrm{a})$ is the phase portrait when parameters $a=$ $8, b=5, c=3.4, d=60$, and $e=4$ and initial conditions $y(0)=1, z(0)=1$, and $w(0)=2$ are given. It can be seen from the figure that the system has the coexistence of onewing period-1, two-wing multiperiod, and four-wing multiperiod phenomena. Figure $12(\mathrm{~b})$ is the phase portrait with the given parameters $a=8, b=5, c=15.32, d=60$, and $e=4$ and initial conditions $x(0)=1, z(0)=1$, and $w(0)=2$. It can be seen from the figure that the system has 


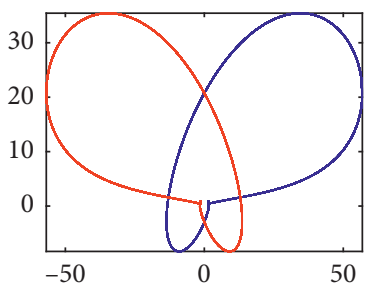

(a)

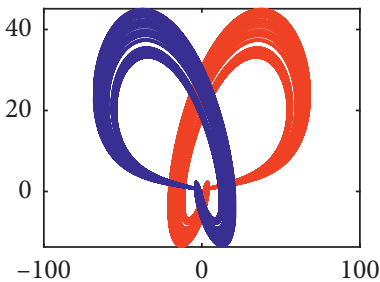

(d)

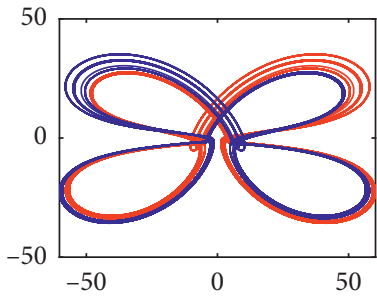

(g)

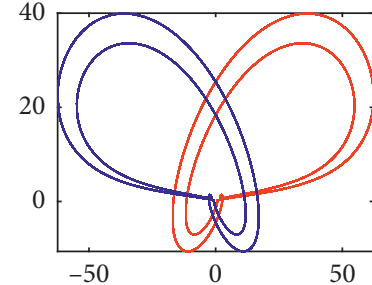

(b)

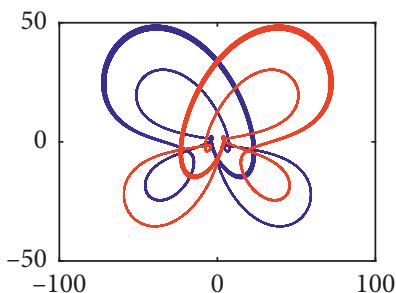

(e)

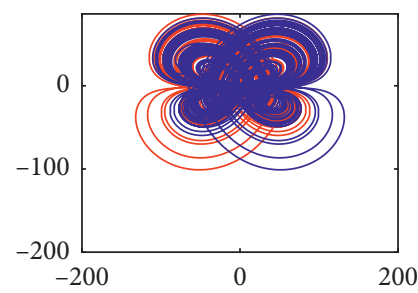

(h)

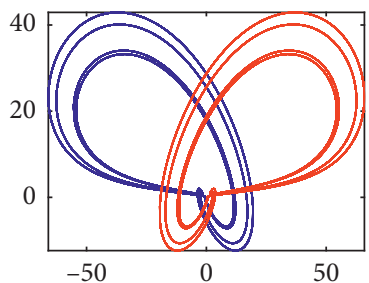

(c)

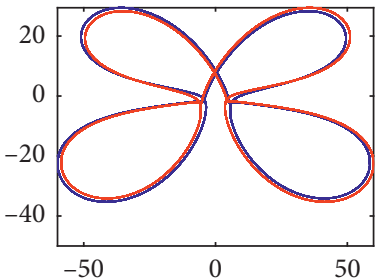

(f)

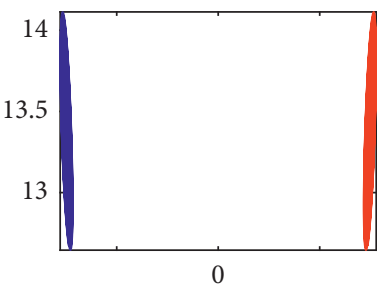

(i)

FIGURE 3: Various coexisting attractors with different values of parameter $a$ in the $y-z$ plane: (a) $a=11.5$, (b) $a=12.3$, (c) $a=12.5$, (d) $a=12.6$, (e) $a=15.8$, (f) $a=20$, (g) $a=19.1$, (h) $a=28$, and (i) $a=31.6$. The blue one is from the initial values $[2,1,1,2]$ and the other from $[-2,-1,1,-2]$.

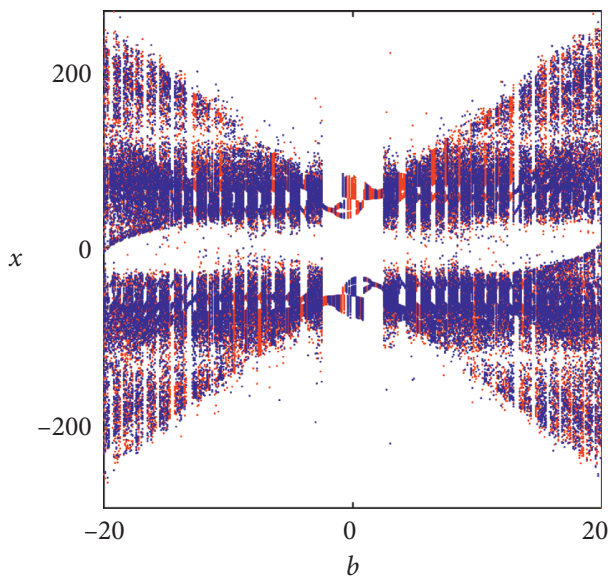

(a)

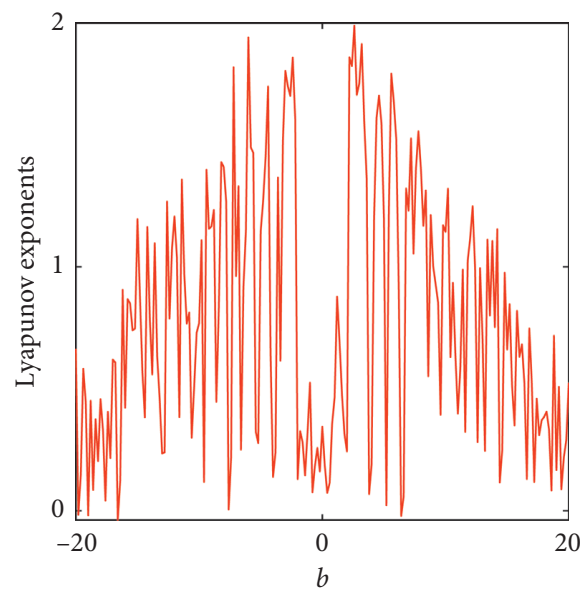

(b)

FIgURE 4: Bifurcation diagram and the largest LE for increasing parameter $b \in[-20,20]$. (a) The bifurcation diagram with initial conditions $[2,1,1,2]$ (blue) and $[-2,-1,1,-2]$ (red). (b) The largest LE.

the coexistence phenomenon of four-wing chaotic attractors and two two-wing chaotic attractors of different directions. It is worth pointing out that the phenomenon of the coexistence polyattractor is very important in cryptography, information security, and secure communication.
3.3. Basin of Attraction. If the attractive regions of chaotic attractors are riddled with each other, a kind of intermittence phenomenon of continuous communication between chaotic attractors will occur when the cross-section Lyapunov exponent is slightly greater than 0 . After the orbit 


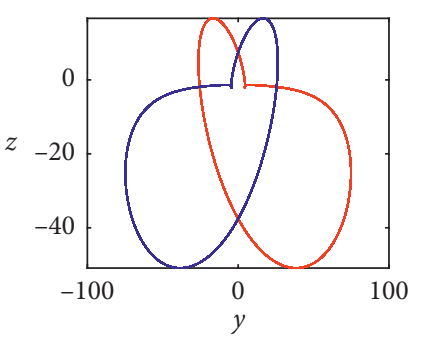

(a)

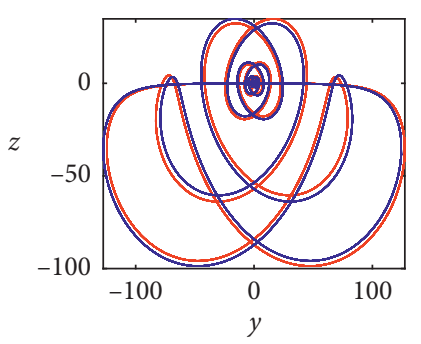

(d)

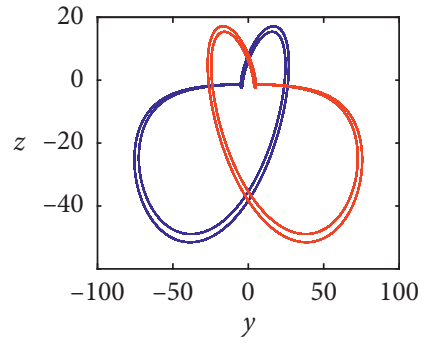

(b)

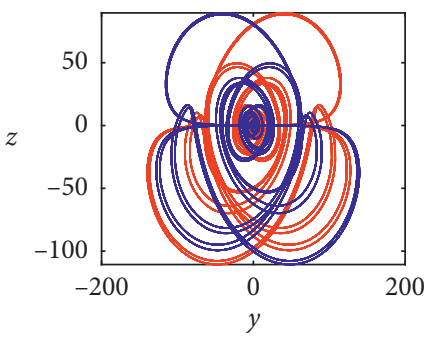

(e)

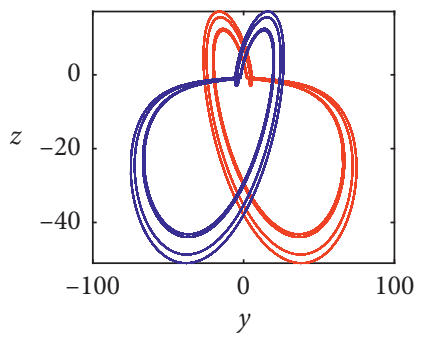

(c)

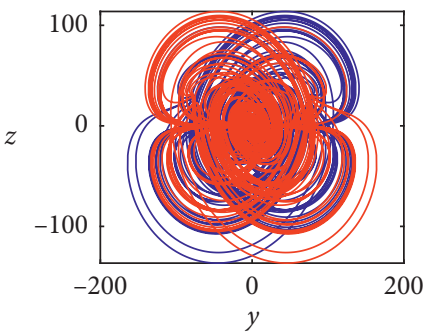

(f)

FiguRE 5: Various coexisting attractors with different values of parameter $b$ in the $y-z$ plane: (a) $b=0$, (b) $b=0.5$, (c) $b=0.58$, (d) $b=19.35$, (e) $b=17.8$, and (f) $b=15$. The blue one is from the initial values $[2,1,1,2]$ and the other from $[-2,-1,1,-2]$.

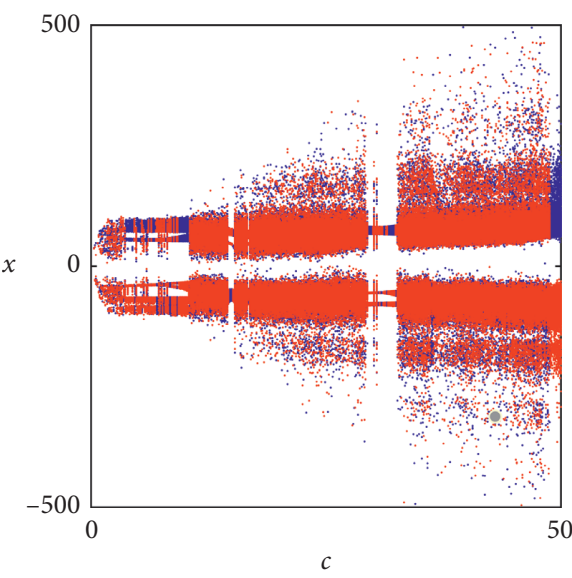

(a)

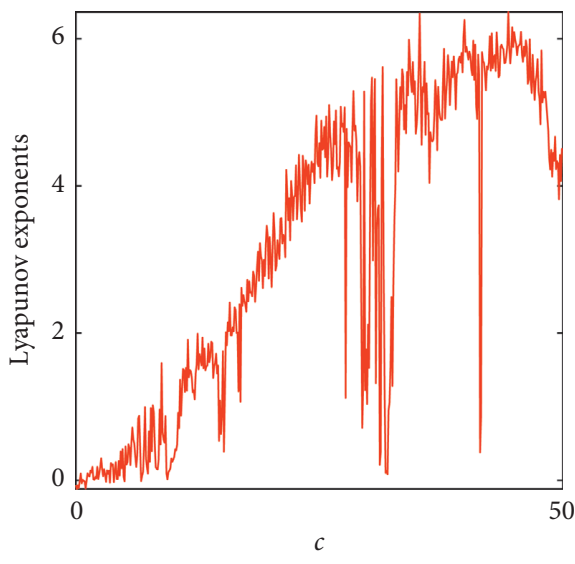

(b)

FIGURE 6: Bifurcation diagram and the largest LE for increasing parameter $c \in[0,50]$. (a) The bifurcation diagram with initial conditions $[2,1,1,2]$ (blue) and $[-2,-1,1,-2]$ (red). (b) The largest LE.

stays near one chaotic attractor for a period of time, it jumps to another chaotic attractor and stays for a period of time, and then repeats the above process [71]. In order to verify whether the sieve domains of the system are mutually riddled, we not only observe the graph directly, but also use the Lyapunov index to judge; for example, according to the parameters and initial conditions of the four-wing chaotic system (1), we calculate that the second Lyapunov exponent is $\lambda_{2}=0.0014$, indicating that the system is mutually riddled.

The Wada domain is possible when the system has three or more attractors $[72,73]$. When studying the formation mechanism of the Wada domain, Nusse and Yorke introduced the concept of the trap region and the domain cell [74]. There are domain cells in the Wada domain, but there are no trap regions and domain cells in the global sieve domain of this system because every point in the global sieve domain has points in its neighborhood that belong to other attractive domains [75]. Figure 13 is the basin of attraction of system (1) with respect to $x(0)-y(0)$ and $z(0)-w(0)$ planes, respectively. It can be shown that there is no open set in this sieve domain. Therefore, we believe that the sieve domain of the system is formed by the local-global bifurcation of the sieve domain.

3.4. Transient Chaos. Transient chaos is a very common phenomenon in nonlinear dynamic systems. When unattractive saddle points appear in the phase space, the system will be chaotic within a certain time range. With the development of time, the system will eventually become 


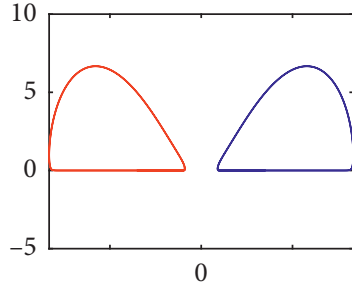

(a)

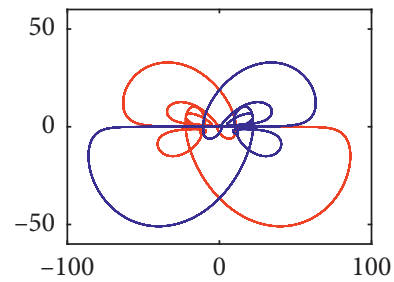

(e)

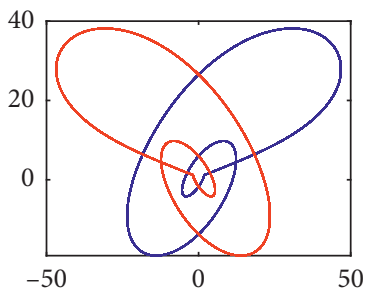

(i)

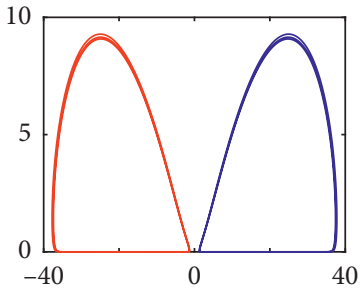

(b)

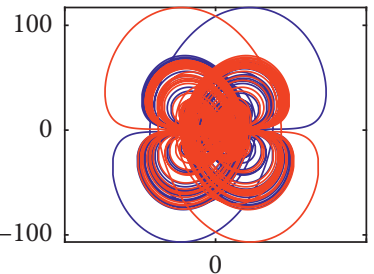

(f)

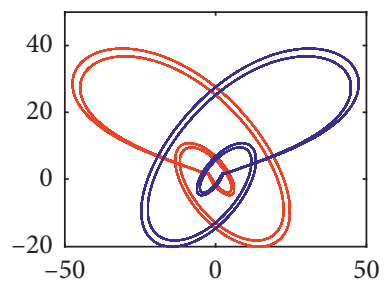

(j)

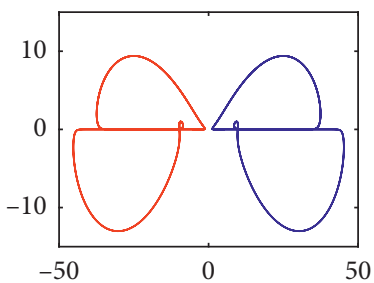

(c)

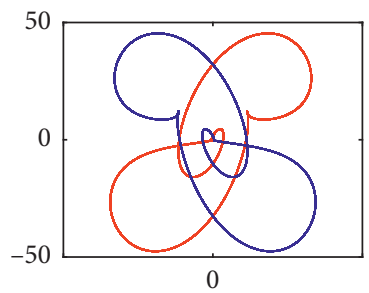

(g)

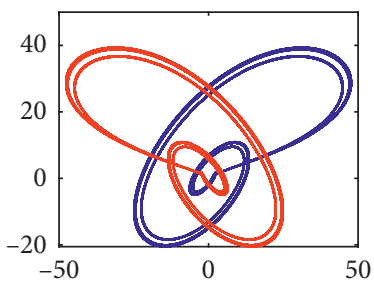

(k)

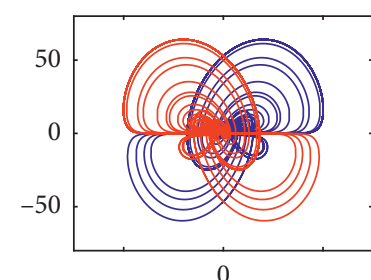

(d)

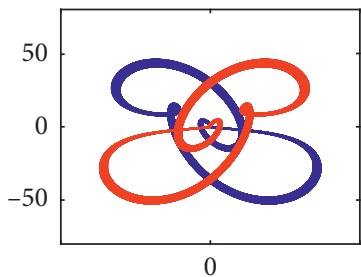

(h)

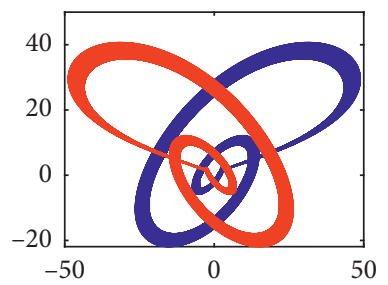

(l)

FIGURE 7: Various coexisting attractors with different values of parameter $c$ in the $y-z$ plane: (a) $c=0.06$, (b) $c=0.1,(\mathrm{c}) c=0.2$, (d) $c=3.4$, (e) $c=3.5$, (f) $c=10.6$, (g) $c=15$, (h) $c=15.32$, (i) $c=31$, (j) $c=31.8$, (k) $c=31.9$, and (l) $c=32.5$. The blue one is from the initial values $[2,1,1,2]$ and the other from $[-2,-1,1,-2]$.

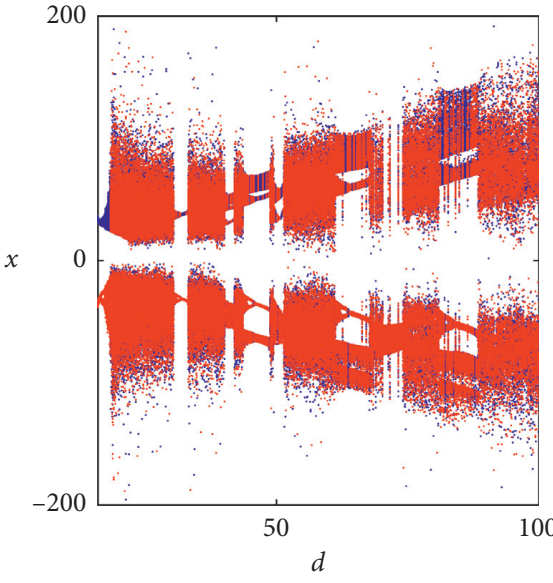

(a)

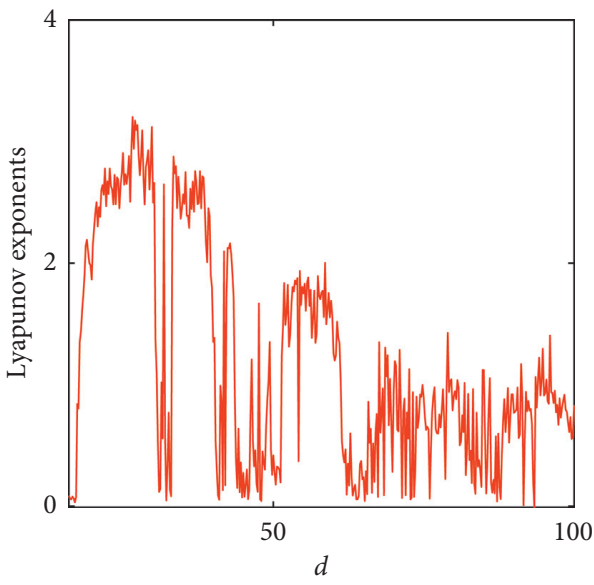

(b)

FIGURE 8: Bifurcation diagram and the largest LE for increasing parameter $d \in[16,100]$. (a) The bifurcation diagram with initial conditions $[2,1,1,2]$ (blue) and $[-2,-1,1,-2]$ (red). (b) The largest LE.

nonchaotic, which is called transient chaos [40]. As shown in Figure 14, under parameters $a=8, b=5, c=12, d=17.5$, and $e=4$ and initial values $[2,1,1,2]$, the transient chaos phenomenon is generated in system (1), and the trajectory of the system evolves over time from transient chaos to steady-state multiperiod behavior. Figure $14(\mathrm{a})$ is the time-domain waveform of $t \in[0,200]$. It can be seen from Figure 14(a) that the system is in a chaotic state in $t \in[0,100]$ and a periodic state in $t \in[100,200]$. Figures 14(b) and 14(c) are the phase portraits of transient chaotic and steady-state periodic states, respectively. Figure 15 is the transient chaos phenomenon under the parameters $a=8, b=5, c=12, d=60$, and $e=11.8$ and the initial values $[2,1,1,2]$. Different from Figure 14 , the system 


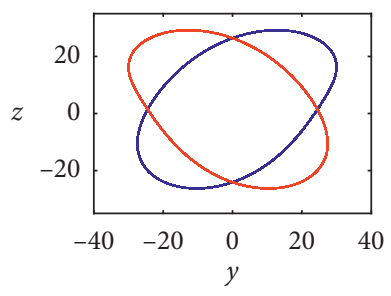

(a)

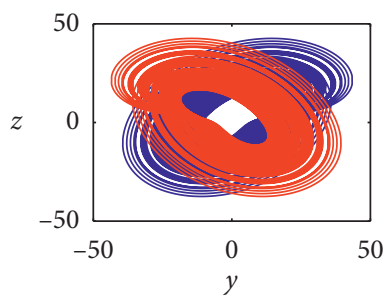

(d)

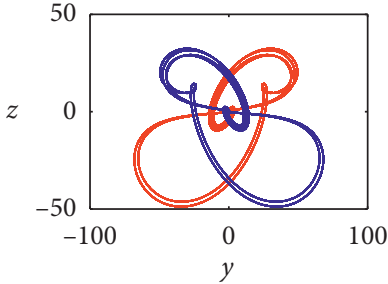

(g)

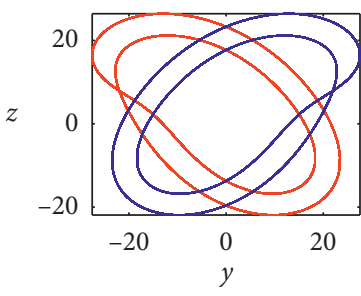

(b)

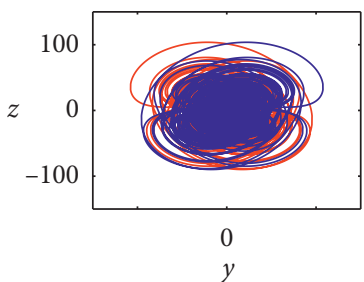

(e)

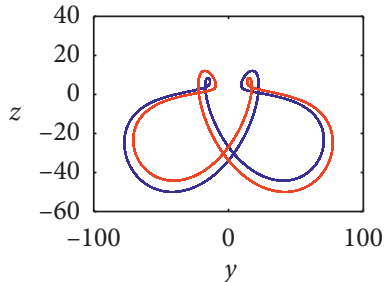

(h)

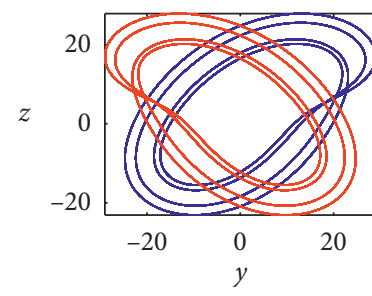

(c)

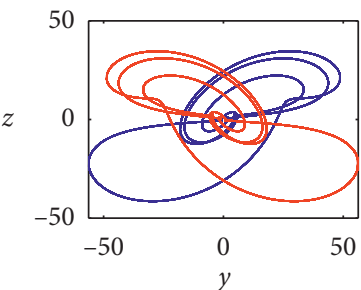

(f)

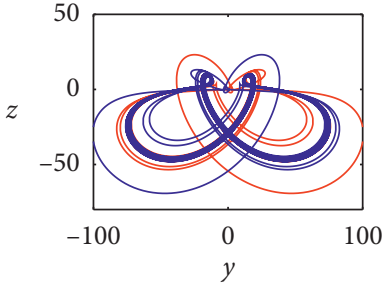

(i)

FIGURE 9: Various coexisting attractors with different values of parameter $d$ in the $y-z$ plane, (a) $d=16,(\mathrm{~b}) d=17$, (c) $d=17.2$, (d) $d=17.8$, (e) $d=25$, (f) $d=42$, (g) $d=50$, (h) $d=68$, and (i) $d=70$. The blue one is from the initial values $[2,1,1,2]$ and the other from $[-2,-1,1,-2]$.

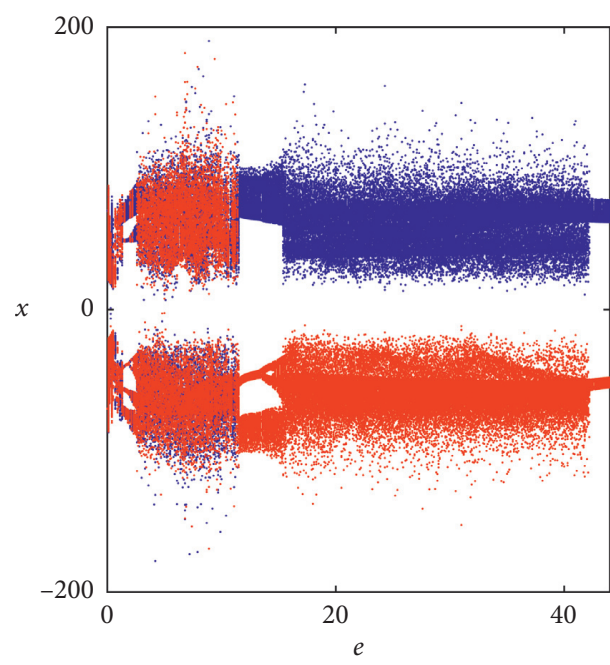

(a)

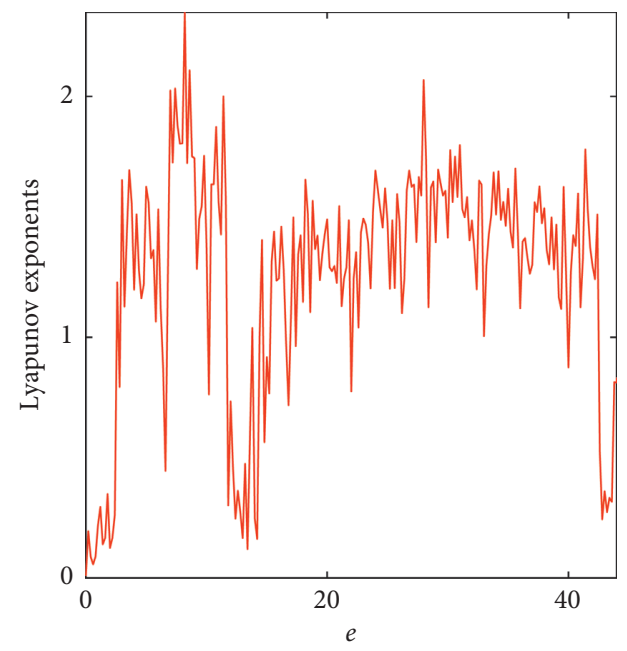

(b)

FIGURE 10: Bifurcation diagram and the largest LE for increasing parameter $e \in[0,44]$. (a) The bifurcation diagram with initial conditions $[2,1,1,2]$ (blue) and $[-2,-1,1,-2]$ (red). (b) The largest LE.

has two different transient chaos states with the evolution of time, as shown in Figures 15(b) and 15(c). These two transient chaos states are both two-wing chaotic attractors, but the direction track of entering chaos is different. Figure $15(\mathrm{~d})$ is the phase portrait of the steadystate period-1.
3.5. Offset Boosting. In this section, we will discuss the offset boosting control. There are generally two ways to construct offset boosting: (1) when a variable appears only once in a nonlinear system, adding a constant to the variable will produce an offset; (2) if every variable in a nonlinear system occurs more than once, it is necessary to construct a variable 


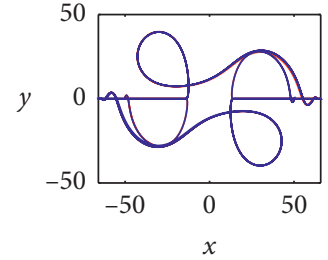

(a)

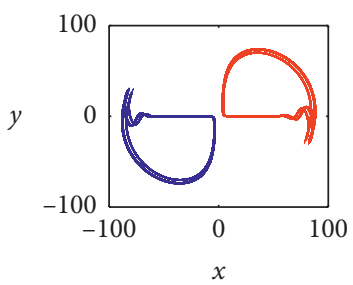

(d)

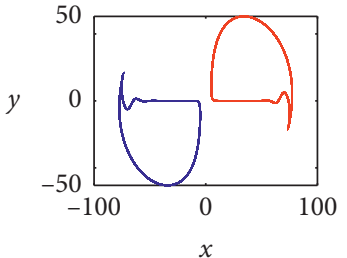

(b)

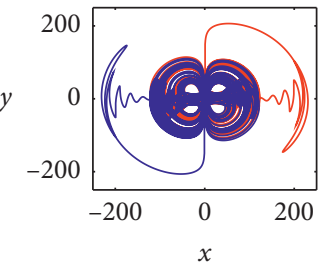

(e)

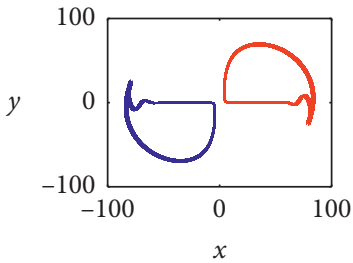

(c)

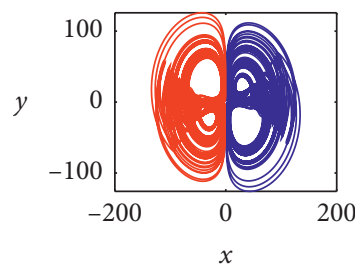

(f)

Figure 11: Various coexisting attractors with different values of parameter $e$ in the $x-y$ plane, (a) $e=0.2,(\mathrm{~b}) e=1.5,(\mathrm{c}) e=2,(\mathrm{~d}) e=2.1$, (e) $e=5$, and (f) $e=17$. The blue one is from the initial values $[2,1,1,2]$ and the other from $[-2,-1,1,-2]$.

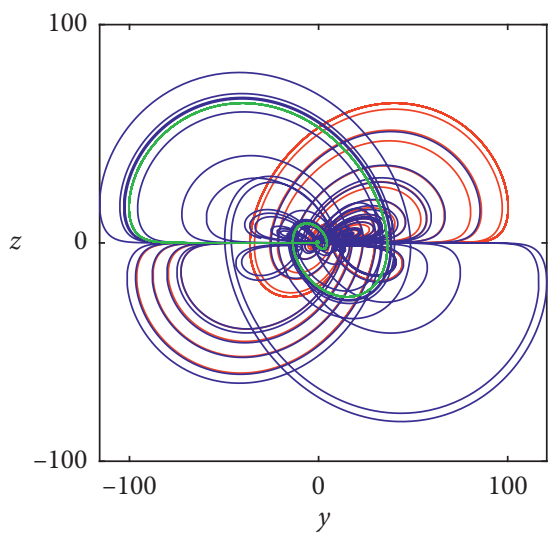

(a)

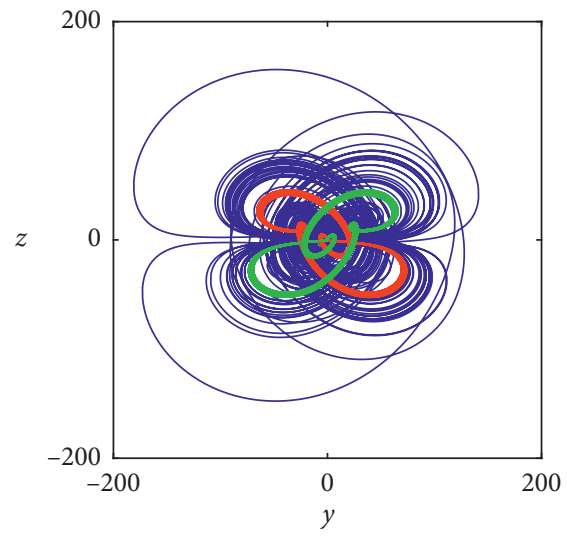

(b)

FiguRe 12: Phase portraits of coexisting multiple attractors. (a) $x(0)=2$ (red), $x(0)=0.2$ (green), and $x(0)=6.5$ (blue); (b) $y(0)=0.0001$ (blue), $y(0)=1$ (red), and $y(0)=8$ (green).

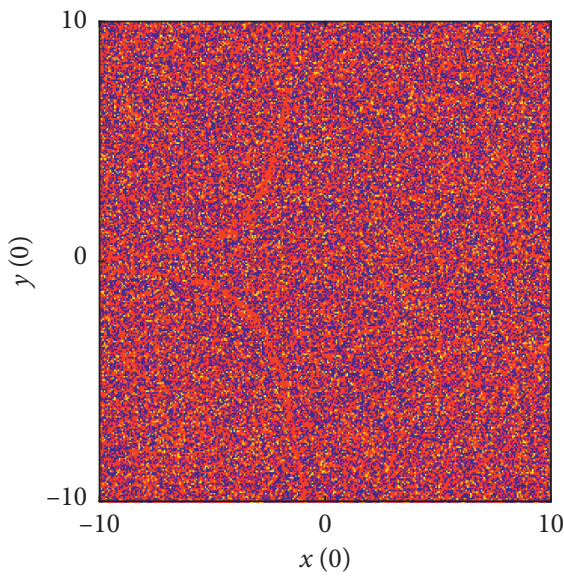

(a)

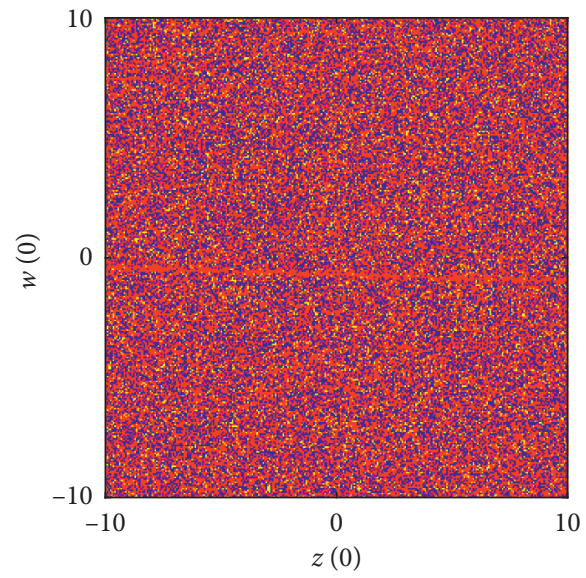

(b)

Figure 13: The basin of attraction of system (1): (a) in the $x(0)-y(0)$ plane for $z(0)=1$ and $w(0)=2$; (b) in the $z(0)-w(0)$ plane for $x(0)=2$ and $y(0)=1$. 


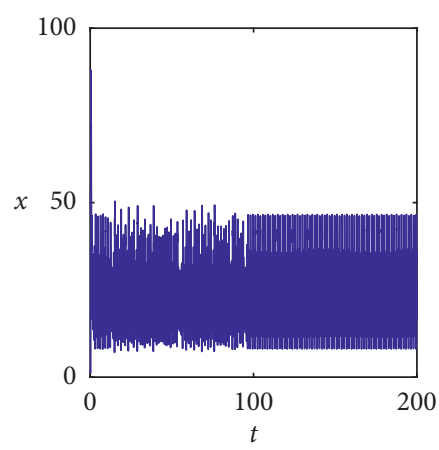

(a)

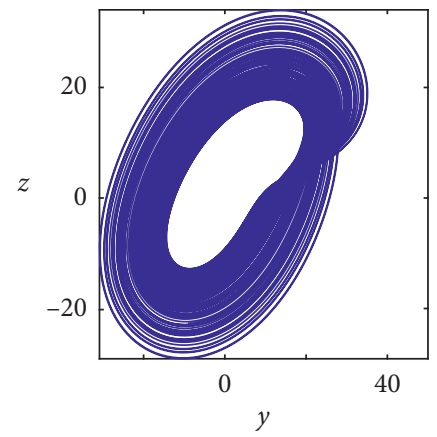

(b)

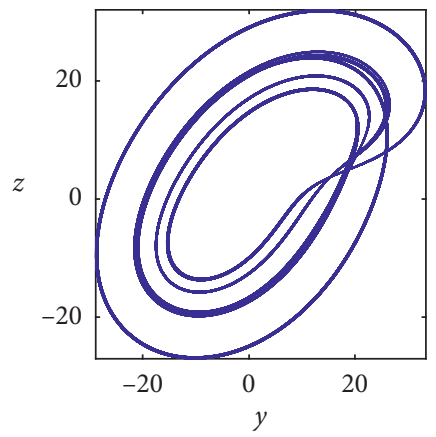

(c)

Figure 14: Transient chaos and steady-state multiperiod. (a) Time-domain waveform of $x$ in the time interval [0,200]. (b) Phase portrait of the transient single-wing chaotic attractor in the time interval $[0,100]$. (c) Phase portrait of the steady-state multiperiod in the time interval $[100,200]$.

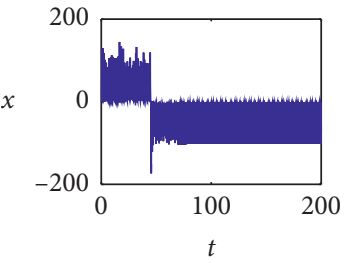

(a)

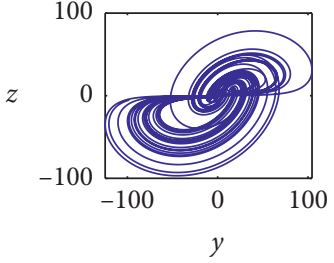

(b)

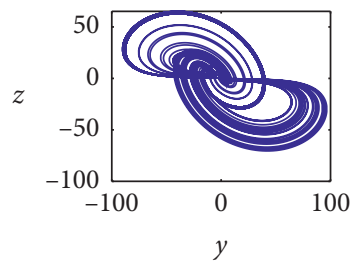

(c)

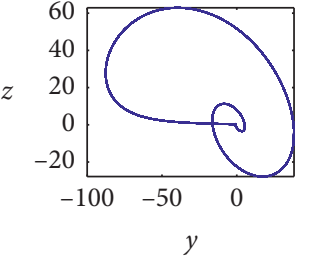

(d)

Figure 15: Transient chaos and steady-state period-1. (a) Time-domain waveform of $x$ in the time interval [0,200]. (b) Phase portrait of the transient two-wing chaotic attractor in the time interval $[0,45)$. (c) Phase portrait of the transient two-wing chaotic attractor in the time interval $[45,700]$. (d) Phase portrait of the steady-state period-1 in the time interval $[70,200]$.

that occurs once by some means [45]. Since every variable in system (1) appears more than once, by making the parameter $b=0$ in the first equation, the variable $w$ appears only once in the system, and then the constant term is added after the variable $g$, as shown in the following equation:

$$
\left\{\begin{array}{l}
\dot{x}=-a x+y z, \\
\dot{y}=c y-x z, \\
\dot{z}=x y-d z, \\
\dot{w}=x z-e(w+g) .
\end{array}\right.
$$

Figure 16(a) is the simulation result of phase portraits when system parameters are $a=8, c=12, d=60$, and $e=4$, initial conditions are set as $[2,1,1,2]$, and three different control parameters are $g=-50,0$, and 50. Figure $16(\mathrm{~b})$ is the simulation result of the phase portraits when system parameters are $a=8, c=12, d=30$, and $e=4$, initial conditions are set as $[2,1,1,2]$, and three different control parameters are $g=-70,0$, and 70 . Figure 16 shows that the variable $w$ can be moved from a bipolar signal to a unipolar signal when the control parameter $g$ is changed.

\section{FPGA Implementation of Yu-Wang Chaotic System}

In this section, we implement the $4 \mathrm{D} \mathrm{Yu-Wang}$ four-wing chaotic system on FPGA using the RK4 algorithm and the 32-bit IEEE 754 floating-point standard. The core idea of designing a chaotic signal generator with FPGA is to divide the whole system into several functional modules, which contain many computing units, including multiplier, adder, and subtracter [76]. These running units are created in cooperation with IP core generators and should follow the IEEE 754 standard. The chaotic system design based on the RK4 algorithm is mainly divided into three modules: data selection module, chaotic oscillation module, and data processing module. Data selection module is to obtain the initial conditions of the system. The initial conditions of the first run of the system are defined by the designer himself. The chaotic oscillation module mainly uses the parallel pipeline structure to calculate the RK4 algorithm to generate the output value within a certain clock period and then feeds back the output value to the data selector as the initial value of the next operation. The data processing module converts 32-bit floating-point signals to 14-bit fixed-point signals. The 14-bit output signal is then downloaded to the FPGA development board for synthesis. Finally, the 14-bit digital signal is converted into an analog signal through a digitalanalog converter and displayed on an oscilloscope.

On the Xilinx ZYNQ-XC7Z020 FPGA chip and the digital-to-analog converter AN9767, the digital hardware implementation of the 4D Yu-Wang four-wing oscillator based on the RK4 algorithm is synthesized. The whole design process is calculated, synthesized, and implemented by Vivado 2018.3, synthesized on the FPGA chip, and outputs 


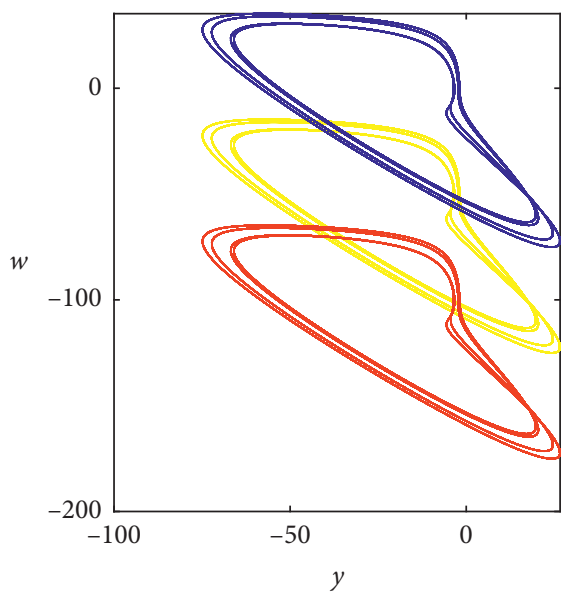

(a)

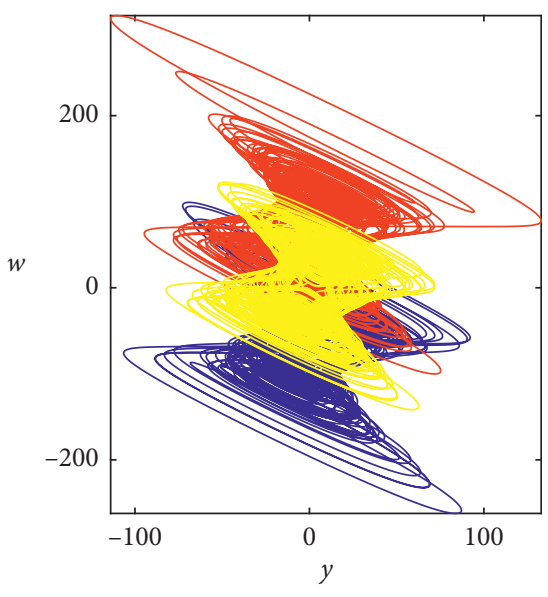

(b)

Figure 16: Offset boosting with control parameter $g$. (a) In the $y-w$ plane for $g=0$ (yellow), $g=50$ (red), and $g=-50$ (blue). (b) In the $y-w$ plane for $g=0$ (yellow), $g=70$ (red), and $g=-70$ (blue).
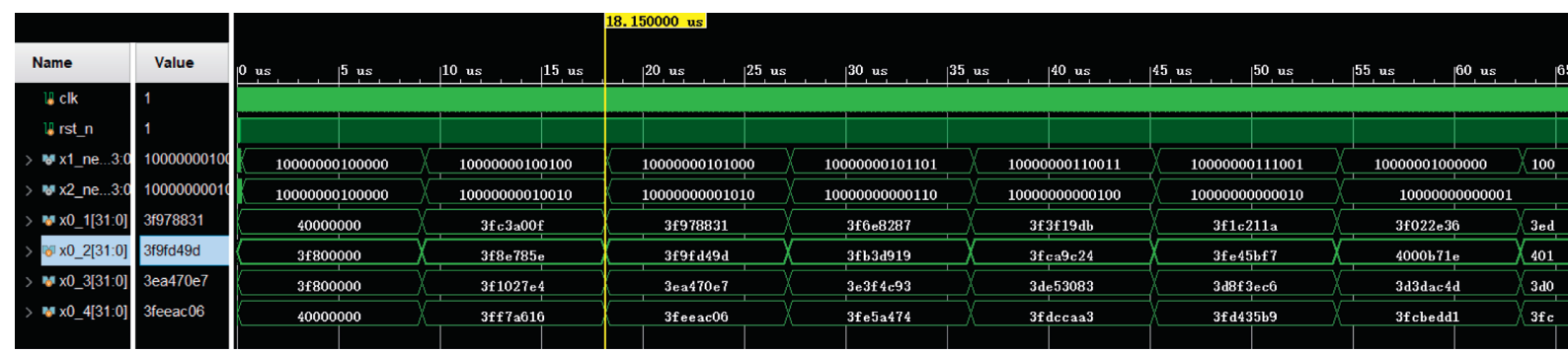

FIGURE 17: Simulation results of the FPGA-based chaotic system with the RK4 algorithm.

TABLE 2: Xilinx ZYNQ-XC7Z020 FPGA chip resource usage.

\begin{tabular}{lccc}
\hline Resource & Used & Available & Utilization (\%) \\
\hline Slice register & 20842 & 106400 & 19.60 \\
Number of slice LUTs & 15931 & 53200 & 29.99 \\
Number of bonded IOBs & 34 & 125 & 27.2 \\
Number of BUFG & 1 & 32 & 3.13 \\
Max. clock frequency & $161.212 \mathrm{MHz}$ & - & - \\
\hline
\end{tabular}

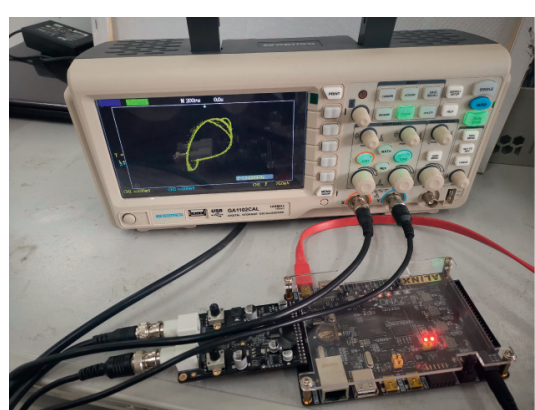

(a)

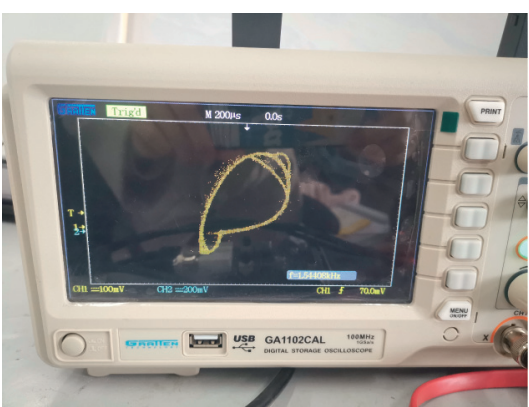

(b)

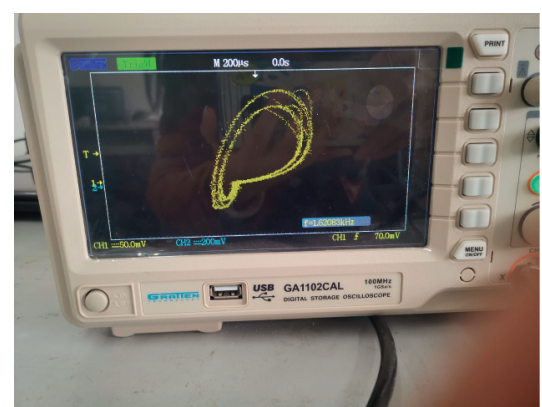

(c)

Figure 18: Continued. 


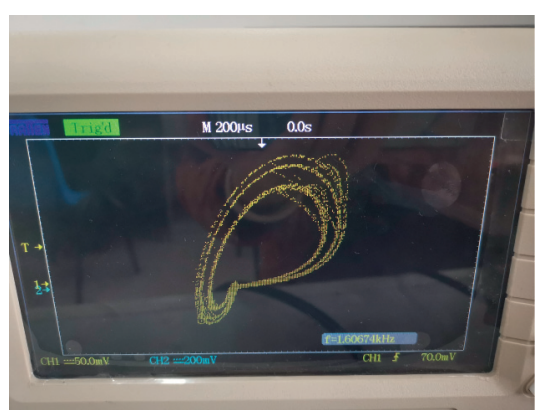

(d)

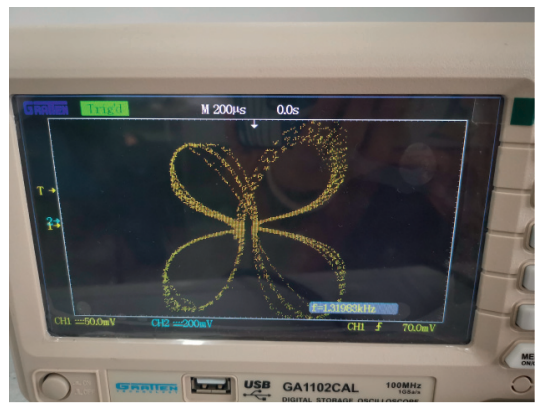

(g)

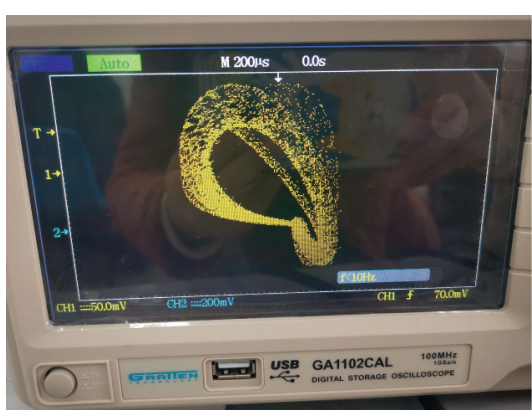

(e)

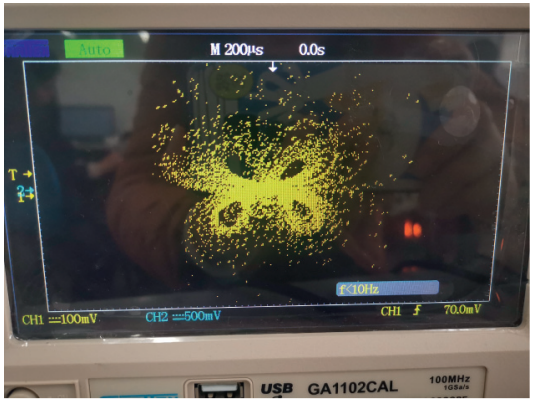

(h)

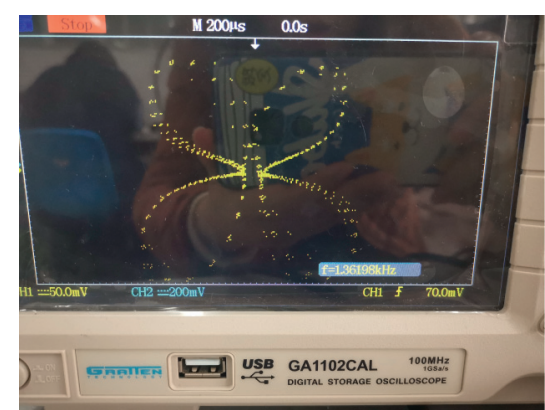

(f)

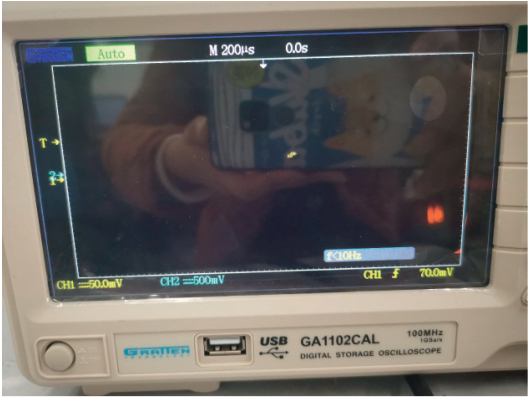

(i)

FIGURE 18: The phase diagrams of the 4D Yu-Wang four-wing chaotic system realized on FPGA based on the RK4 algorithm. (a) Simulation panorama; (b) one-wing period-1; (c) one-wing period-2; (d) one-wing period-4; (e) one-wing chaotic attractor; (f) four-wing period-1; (g) four-wing period-4; (h) four-wing chaotic attractor; (i) stable state.

the analog signal through the digital-to-analog converter and displayed on an oscilloscope. In the design process, the 32-bit IEEE 754 floating-point standard is adopted, but in order to better analyze the experimental results, we convert 32-bit data into 16-bit data. Figure 17 is the discrete sequence generated by the chaos oscillator based on FPGA. Table 2 shows the statistical data of the Yu-Wang system implemented on the Xilinx ZYNQ-XC7Z020 FPGA chip. It can be seen from the table that the maximum operating frequency of the designed chaotic oscillator based on FPGA is $161.212 \mathrm{MHz}$. Figure 18 shows the phase diagrams of the $4 \mathrm{D} \mathrm{Yu-Wang}$ four-wing chaotic system displayed on the oscilloscope, which are consistent with the attractor phase portraits shown in MATLAB simulation of Figure 3, which proves that the method of realizing the chaotic system with FPGA is reliable.

\section{Conclusion}

Our objective is to further study the $4 \mathrm{D} \mathrm{Yu}$-Wang four-wing chaotic system using standard nonlinear analysis tools such as Lyapunov exponential spectrum, bifurcation diagram, basin of attraction, and phase portraits. The simulation results show that the system has very complex dynamical behaviors, including coexistence attractor, multistability, transient chaos, and offset boosting. It is worth noting that different system parameters have different coexistence multiple attractors. At the same time, it can also generate transient one-wing chaotic attractors and transient twowing chaotic attractors. Secondly, using the Xilinx ZYNQXC7Z020 FPGA chip and the digital-to-analog converter
AN9767, the phase diagram of the Yu-Wang chaotic system was displayed on the oscilloscope, including one-wing period-1, one-wing period-2, one-wing period-4, one-wing chaotic attractor, four-wing period-1, four-wing period-4, four-wing chaotic attractor, and steady state. This paper proves that the $4 \mathrm{D} \mathrm{Yu}$-Wang four-wing chaotic system based on the FPGA design can be applied to various embedded engineering system applications based on chaos, including cryptographic system, secure communication, and chaotic random number generator.

\section{Data Availability}

All data used to support the findings of this study are available from the corresponding author upon request.

\section{Conflicts of Interest}

The authors declare that they have no conflicts of interest.

\section{Acknowledgments}

This work was supported by the National Natural Science Foundation of China, under Grants 61504013, 61702052, and 61901169, the Natural Science Foundation of Hunan Province, under Grants 2019JJ50648, 2020JJ4622, and 2019JJ40190, the Guangxi Key Laboratory of Cryptography and Information Security, under Grant GCIS201919, the Postgraduate Training Innovation Base Construction Project of Hunan Province, under Grant 2020-172-48, the Postgraduate Scientific Research Innovation Project of Hunan 
Province, under Grant CX20200884, the Scientific Research Fund of Hunan Provincial Education Department, under grant 18A137, and the young teacher development program project of the Changsha University of Science and Technology, under grant 2019QJCZ013.

\section{References}

[1] C. Wang, L. Xiong, J. Sun, and W. Yao, "Memristor-based neural networks with weight simultaneous perturbation training," Nonlinear Dynamics, vol. 95, no. 4, pp. 2893-2906, 2019.

[2] W. Yao, C. Wang, J. Cao, Y. Sun, and C. Zhou, "Hybrid multisynchronization of coupled multistable memristive neural networks with time delays," Neurocomputing, vol. 363, pp. 281-294, 2019.

[3] X. Ma, J. Mou, J. Liu, C. Ma, F. Yang, and X. Zhao, "A novel simple chaotic circuit based on memristor-memcapacitor," Nonlinear Dynamics, vol. 100, no. 3, pp. 2859-2876, 2020.

[4] Y. M. Tan and C. H. Wang, "A simple locally active memristor and its application in HR neurons," Chaos, vol. 30, no. 5, Article ID 053118, 2020.

[5] F. Yu, Q. Wan, J. Jin et al., "Design and FPGA implementation of a pseudorandom number generator based on a four-wing memristive hyperchaotic system and Bernoulli map," IEEE Access, vol. 7, pp. 181884-181898, 2019.

[6] F. Yu, L. Li, Q. Tang, S. Cai, Y. Song, and Q. Xu, "A survey on true random number generators based on chaos," Discrete Dynamics in Nature and Society, vol. 2019, Article ID 2545123 , 10 pages, 2019.

[7] F. Yu, L. Liu, S. Qian et al., "Chaos-based application of a novel multistable 5D memristive hyperchaotic system with coexisting multiple attractors," Complexity, vol. 2020, Article ID 8034196, 19 pages, 2020.

[8] Q. Zhao, C. H. Wang, and X. Zhang, "A universal emulator for memristor, memcapacitor, and meminductor and its chaotic circuit," Chaos, vol. 29, Article ID 013141, 2019.

[9] F. Yu, H. Shen, L. Liu et al., "CCII and FPGA realization: a multistable modified fourth-order autonomous chua's chaotic system with coexisting multiple attractors," Complexity, vol. 2020, Article ID 5212601, 17 pages, 2020.

[10] F. Yu, L. Liu, H. Shen et al., "Dynamic analysis, circuit design and synchronization of a novel 6D memristive four-wing hyperchaotic system with multiple coexisting attractors," Complexity, vol. 2020, Article ID 5904607, 17 pages, 2020.

[11] G. F. Cheng, C. H. Wang, and H. A. Chen, "Novel color image encryption algorithm based on hyperchaotic system and permutation-diffusion architecture," International Journal of Bifurcation and Chaos, vol. 29, Article ID 1950115, 2019.

[12] Y. Chen, W. Xu, J. Zuo, and K. Yang, "The fire recognition algorithm using dynamic feature fusion and IV-SVM classifier," Cluster Computing, vol. 22, no. S3, pp. 7665-7675, 2019.

[13] F. Peng, X. W. Zhu, and M. Long, "An ROI privacy protection scheme for H.264 video based on FMO and chaos," IEEE Transactions on Information Forensics and Security, vol. 8, no. 10 , pp. $1688-1699,2013$.

[14] S. Wang, C. Wang, and C. Xu, "An image encryption algorithm based on a hidden attractor chaos system and the Knuth-Durstenfeld algorithm," Optics and Lasers in Engineering, vol. 128, Article ID 105995, 2020.

[15] F. Yu, L. Liu, L. Xiao, K. Li, and S. Cai, "A robust and fixedtime zeroing neural dynamics for computing time-variant nonlinear equation using a novel nonlinear activation function," Neurocomputing, vol. 350, pp. 108-116, 2019.

[16] J. Jin, L. Zhao, M. Li, F. Yu, and Z. Xi, "Improved zeroing neural networks for finite time solving nonlinear equations," Neural Computing and Applications, vol. 32, no. 9, pp. 4151-4160, 2020.

[17] L. Zhou, F. Tan, F. Yu, and W. Liu, "Cluster synchronization of two-layer nonlinearly coupled multiplex networks with multi-links and time-delays," Neurocomputing, vol. 359, pp. 264-275, 2019.

[18] H. R. Lin and C. H. Wang, "Influences of electromagnetic radiation distribution on chaotic dynamics of a neural network," Applied Mathematics and Computation, vol. 369, Article ID 124840, 2020.

[19] Y. Sun, C. Zhou, H. Lin et al., "Synchronization of inertial memristive neural networks with time-varying delays via static or dynamic event-triggered control," Neurocomputing, vol. 404, pp. 367-380, 2020.

[20] F. Yu, L. Gao, K. Gu, B. Yin, Q. Wan, and Z. Zhou, "A fully qualified four-wing four-dimensional autonomous chaotic system and its synchronization," Optik, vol. 131, pp. 79-88, 2017.

[21] Y. Huang, L. Huang, Y. Wang, Y. Peng, and F. Yu, "Shape synchronization in driver-response of 4 -D chaotic system and its application in image encryption," IEEE Access, vol. 8, pp. 135308-135319, 2020.

[22] C. Zhou, C. H. Wang, Y. C. Sun, and W. Yao, "Weighted sum synchronization of memristive coupled neural networks," Neurocomputing, vol. 403, pp. 225-232, 2020.

[23] F. Yu and Y. Song, "Complete switched generalized function projective synchronization of a class of hyperchaotic systems with unknown parameters and disturbance inputs," Journal of Dynamic Systems, Measurement, and Control-Transactions of the ASME, vol. 136, no. 1, Article ID 014505, 2014.

[24] M. Long, Y. Chen, and F. Peng, "Simple and accurate analysis of BER performance for DCSK chaotic communication," IEEE Communications Letters, vol. 15, no. 11, pp. 1175-1177, 2011.

[25] F. Yu, S. Qian, X. Chen et al., "A new 4D four-wing memristive hyperchaotic system: dynamical analysis, electronic circuit design, shape synchronization and secure communication," International Journal of Bifurcation and Chaos, vol. 30, no. 10, Article ID 2050147, 2020.

[26] F. Yu, L. Liu, B. He et al., "Analysis and FPGA realization of a novel 5D hyperchaotic four-wing memristive system, active control synchronization, and secure communication application," Complexity, vol. 2019, Article ID 4047957, 18 pages, 2019.

[27] F. Yu, Z. Zhang, L. Liu et al., "Secure communication scheme based on a new 5D multistable four-wing memristive hyperchaotic system with disturbance inputs," Complexity, vol. 2020, Article ID 5859273, 16 pages, 2020.

[28] L. Zhou, F. Tan, and F. Yu, "A robust synchronization-based chaotic secure communication scheme with double-layered and multiple hybrid networks," IEEE Systems Journal, vol. 14, no. 2, pp. 2508-2519, 2020.

[29] Q. L. Deng, C. H. Wang, and L. Yang, "Four-wing hidden attractors with one stable equilibrium point," International Journal of Bifurcation and Chaos, vol. 30, no. 6, Article ID 2050086, 2020.

[30] L. Zhou, C. Wang, and L. Zhou, "Generating hyperchaotic multi-wing attractor in a 4D memristive circuit," Nonlinear Dynamics, vol. 85, no. 4, pp. 2653-2663, 2016.

[31] F. Yu, P. Li, K. Gu, and B. Yin, "Research progress of multiscroll chaotic oscillators based on current-mode devices," Optik, vol. 127, no. 13, pp. 5486-5490, 2016. 
[32] F. Yu, C. Wang, and H. He, "Grid multiscroll hyperchaotic attractors based on colpitts oscillator mode with controllable grid gradient and scroll numbers," Journal of Applied Research and Technology, vol. 11, no. 3, pp. 371-380, 2013.

[33] Q. L. Deng and C. H. Wang, "Multi-scroll hidden attractors with two stable equilibrium points," Chaos: An Interdisciplinary Journal of Nonlinear Science, vol. 29, no. 9, Article ID 093112, 2019.

[34] X. Zhang, C. Wang, W. Yao, and H. Lin, "Chaotic system with bondorbital attractors," Nonlinear Dynamics, vol. 97, no. 4, pp. 2159-2174, 2019.

[35] F. Yang, J. Mou, J. Liu et al., "Characteristic analysis of the fractional-order hyperchaotic complex system and its image encryption application," Signal Processing, vol. 169, Article ID 107373, 2020.

[36] X. Ye, J. Mou, C. Luo, and Z. Wang, "Dynamics analysis of Wien-bridge hyperchaotic memristive circuit system," Nonlinear Dynamics, vol. 92, no. 3, pp. 923-933, 2018.

[37] C. Xu, J. Sun, and C. Wang, "An image encryption algorithm based on random walk and hyperchaotic systems," International Journal of Bifurcation and Chaos, vol. 30, no. 4, Article ID 2050060, 2020.

[38] M. Zhou and C. Wang, "A novel image encryption scheme based on conservative hyperchaotic system and closed-loop diffusion between blocks," Signal Processing, vol. 171, Article ID 107484, 2020.

[39] X. Zhang and C. H. Wang, "Multiscroll hyperchaotic system with hidden attractors and its circuit implementation," International Journal of Bifurcation and Chaos, vol. 29, Article ID 1950117, 2019.

[40] H. Lin, C. Wang, and Y. Tan, "Hidden extreme multistability with hyperchaos and transient chaos in a Hopfield neural network affected by electromagnetic radiation," Nonlinear Dynamics, vol. 99, no. 3, pp. 2369-2386, 2020.

[41] B. Bao, A. Hu, H. Bao, Q. Xu, M. Chen, and H. Wu, "Threedimensional memristive hindmarsh-rose neuron model with hidden coexisting asymmetric behaviors," Complexity, vol. 2018, Article ID 3872573, 11 pages, 2018.

[42] L. Cui, M. Lu, Q. Ou et al., "Analysis and circuit implementation of fractional order multi-wing hidden attractors," Chaos, Solitons \& Fractals, vol. 138, Article ID 109894, 2020.

[43] H. Lin, C. Wang, Y. Sun, and W. Yao, "Firing multistability in a locally active memristive neuron model," Nonlinear Dynamics, vol. 100, pp. 3667-3683, 2020.

[44] V. R. Folifack Signing, J. Kengne, and L. K. Kana, "Dynamic analysis and multistability of a novel four-wing chaotic system with smooth piecewise quadratic nonlinearity," Chaos, Solitons \& Fractals, vol. 113, pp. 263-274, 2018.

[45] A. Bayani, K. Rajagopal, A. J. M. Khalaf, S. Jafari, G. D. Leutcho, and J. Kengne, "Dynamical analysis of a new multistable chaotic system with hidden attractor: antimonotonicity, coexisting multiple attractors, and offset boosting," Physics Letters A, vol. 383, no. 13, pp. 1450-1456, 2019.

[46] A. T. Azar, N. M. Adele, K. S. T. Alain, R. Kengne, and F. H. Bertrand, "Multistability analysis and function projective synchronization in relay coupled oscillators," Complexity, vol. 2018, Article ID 3286070, 12 pages, 2018.

[47] B. A. Mezatio, M. T. Motchongom, B. R. Wafo Tekam, R. Kengne, R. Tchitnga, and A. Fomethe, "A novel memristive 6D hyperchaotic autonomous system with hidden extreme multistability," Chaos, Solitons \& Fractals, vol. 120, pp. 100-115, 2019.
[48] H. Bao, T. Jiang, K. Chu et al., "Memristor-based canonical chua's circuit: extreme multistability in voltage-current domain and its controllability in flux-charge domain," Complexity, vol. 2018, Article ID 5935637, 13 pages, 2018.

[49] X. Zhang and Z. Li, "Hidden extreme multistability in a novel $4 \mathrm{D}$ fractional-order chaotic system," International Journal of Non-linear Mechanics, vol. 111, pp. 14-27, 2019.

[50] H. Lin, C. Wang, and Y. Tan, "Hidden extreme multistability with hyperchaos and transient chaos in a Hopfield neural network affected by electromagnetic radiation," Nonlinear Dynamics, vol. 99, pp. 2369-2386, 2020.

[51] J. Jin, "Programmable multi-direction fully integrated chaotic oscillator," Microelectronics Journal, vol. 75, pp. 27-34, 2018.

[52] J. Jin and L. V. Zhao, "Low voltage low power fully integrated chaos generator," Journal of Circuits, Systems and Computers, vol. 27, Article ID 1850155, 2018.

[53] J. Jin and L. Cui, "Fully integrated memristor and its application on the scroll-controllable hyperchaotic system," Complexity, vol. 2019, Article ID 4106398, 8 pages, 2019.

[54] F. Yu, L. Gao, L. Liu, S. Qian, S. Cai, and Y. Song, "A 1 V, $0.53 \mathrm{~ns}, 59 \mu \mathrm{W}$ current comparator using standard $0.18 \mu \mathrm{m}$ CMOS technology," Wireless Personal Communications, vol. 111, no. 2, pp. 843-851, 2020.

[55] F. Yu, Q. Tang, W. Wang, and H. Wu, “A. 2.A 2.7 GHz lowphase-noise LC-QVCO using the gate-modulated coupling technique," Wireless Personal Communications, vol. 86, no. 2, pp. 671-681, 2016.

[56] F. Yu, "A low-voltage and low-power 3-GHz CMOS LC VCO for S-band wireless applications," Wireless Personal Communications, vol. 78, no. 2, pp. 905-914, 2014.

[57] X. Wu, W. Tan, and $\mathrm{H}$. Wang, "Initial value determination of chua system with hidden attractors and its DSP implementation," Complexity, vol. 2020, Article ID 7638243, 9 pages, 2020.

[58] S. He, K. Sun, H. Wang, X. Mei, and Y. Sun, "Generalized synchronization of fractional-order hyperchaotic systems and its DSP implementation," Nonlinear Dynamics, vol. 92, no. 1, pp. 85-96, 2018.

[59] M. S. Azzaz, C. Tanougast, S. Sadoudi, R. Fellah, and A. Dandache, "A new auto-switched chaotic system and its FPGA implementation," Communications in Nonlinear Science and Numerical Simulation, vol. 18, no. 7, pp. 1792-1804, 2013.

[60] E. Tlelo-Cuautle, L. G. de la Fraga, V.-T. Pham, C. Volos, S. Jafari, and A. d. J. Quintas-Valles, "Dynamics, FPGA realization and application of a chaotic system with an infinite number of equilibrium points," Nonlinear Dynamics, vol. 89, no. 2, pp. 1129-1139, 2017.

[61] B. Atiyeh, J. M. Ali, and R. Karthikeyan, "A novel fractionalorder chaotic system with specific topology: from proposing to FPGA implementation," The European Physical Journal Special Topics, vol. 226, pp. 3729-3745, 2017.

[62] J. X. Yong, X. Cheng, and G. Tao-Tao, "Dynamical analysis and FPGA implementation of a large range chaotic system with coexisting attractors," Results in Physics, vol. 11, pp. 368-376, 2018.

[63] A. Karthikeyan and K. Rajagopal, "FPGA implementation of fractional-order discrete memristor chaotic system and its commensurate and incommensurate synchronisations," Pramana, vol. 90, no. 1, 2018.

[64] Y. W. Guang, L. B. Xu, and L. W. Zhong, "Design and FPGA Implementation of a new hyperchaotic system," Chinese Physics B, vol. 17, pp. 3596-3602, 2018. 
[65] D. Enzeng, Y. Mingfeng, and Z. Cong, "Dynamics, topological horseshoe analysis, ultimate boundary estimations of a new 4D hyperchaotic system and its FPGA implementation," International Journal of Bifurcation and Chaos, vol. 28, Article ID 1850081, 2018.

[66] J.-L. Zhang, W.-Z. Wang, X.-W. Wang, and Z.-H. Xia, "Enhancing security of FPGA-based embedded systems with combinational logic binding," Journal of Computer Science and Technology, vol. 32, no. 2, pp. 329-339, 2017.

[67] R. Karthikeyan, K. Anitha, and S. A. Kuma, "FPGA implementation of novel fractional-order chaotic systems with two equilibriums and no equilibrium and its adaptive sliding mode synchronization," Nonlinear Dynamics, vol. 87, no. 4, pp. 2281-2304, 2017.

[68] S. Cai, B. He, W. Wang et al., "Soft error reliability evaluation of nanoscale logic circuits in the presence of multiple transient faults," Journal of Electronic Testing, vol. 36, pp. 469-483, 2020.

[69] F. Yu, C. H. Wang, and J. W. Yin, "A 4-D chaos with fully qualified four-wing type," Acta Physica Sinica, vol. 61, Article ID 020506, 2012.

[70] A. Wolf, J. B. Swift, H. L. Swinney, and J. A. Vastano, "Determining Lyapunov exponents from a time series," Physica D: Nonlinear Phenomena, vol. 16, no. 3, pp. 285-317, 1985.

[71] J. Dai, D.-R. He, X.-L. Xu, and C.-K. Hu, "A riddled basin escaping crisis and the universality in an integrate-and-fire circuit," Physica A: Statistical Mechanics and Its Applications, vol. 500, pp. 72-79, 2018.

[72] E. Ott, Chaos in Dynamical Systems, Cambridge University Press, Cambridge, UK, 1993.

[73] H. E. Nusse and J. A. Yorke, "Border-collision bifurcations including "period two to period three" for piecewise smooth systems," Physica D: Nonlinear Phenomena, vol. 57, no. 1-2, pp. 39-57, 1992.

[74] Y. Cao, "A note about Milnor attractor and riddled basin," Chaos, Solitons \& Fractals, vol. 19, no. 4, pp. 759-764, 2004.

[75] K. Sathiyadevi, S. Karthiga, V. K. Chandrasekar, D. V. Senthilkumar, and M. Lakshmanan, "Frustration induced transient chaos, fractal and riddled basins in coupled limit cycle oscillators," Communications in Nonlinear Science and Numerical Simulation, vol. 72, pp. 586-599, 2019.

[76] M. Tuna, M. Alçın, İ. Koyuncu, C. B. Fidan, and İ. Pehlivan, "High speed FPGA-based chaotic oscillator design," Microprocessors and Microsystems, vol. 66, pp. 72-80, 2019. 